\title{
Airway management in a patient with Goldenhar syndrome
}

\author{
S Kajal ${ }^{*}$, S Mukherjee ${ }^{2}$, A Saxena ${ }^{1}$, U Asthana $^{1}$ \\ Senior Resident ${ }^{\prime *}$, Department of Anaesthesiology and critical care, ESI PGIMER, Basaidarapur, New \\ Delhi, India. Assistant Professor ${ }^{2}$, BC Roy Postgraduate Institute of Paediatric Sciences, Kolkata, India.
}

*Corresponding author:shvetakajal20@gmail.com

\begin{abstract}
Goldenhar syndrome involves multiple facial, cardiac, renal and neurological deficits which generally culminate into difficult ventilation and intubation. Various devices have been reported, in successfully securing the airway of these children. In our case we have preferred CMAC laryngoscope in securing the difficult paediatric airway and have found it effective, safe and less cumbersome than the traditional direct laryngoscopy.
\end{abstract}

Keywords: Goldenhar syndrome; difficult intubation; CMAC laryngoscope; LMA; direct laryngoscopy

\section{Introduction}

Goldenhar syndrome, a variant of oculo-auriculovertebral dysplasia is a rare congenital disease. It involves pre auricular appendages, fistulas, epibulbar dermoids, vertebral abnormalities, upper eyelid colobomas, subconjunctival lipomas, ear anomalies, hearing loss, unilateral facial hypoplasia, micrognathia, cleft or high-arched palate, congenital heart disease, renal anomalies and mental retardation. ${ }^{1}$ The male: female preponderance is $3: 2 .^{2}$ This syndrome poses a unique challenge to the anaesthesiologist in terms of difficult mask ventilation and intubation due to facial asymmetry, large head, micrognathia, cleft palate, hemi facial hypoplasia, malocclusion and vertebral anomalies. Moderate to severe cardiac anomalies, mental retardation, secondary respiratory problems in a paediatric patient may further compound the problem. We report the successful airway management in a case of Goldenhar syndrome with video laryngoscope (CMAC laryngoscope).

\section{Case Report}

A 3 year old female child $(12 \mathrm{~kg})$ with Goldenhar syndrome was scheduled for lid reconstruction surgery. The preoperative ophthalmic examination showed upper lid coloboma with epibulbar dermoid involving cornea and cystic lesion in orbital cavity. She had occipital encephalocele, preauricular tags and hemifacial asymmetry (Figure 1).

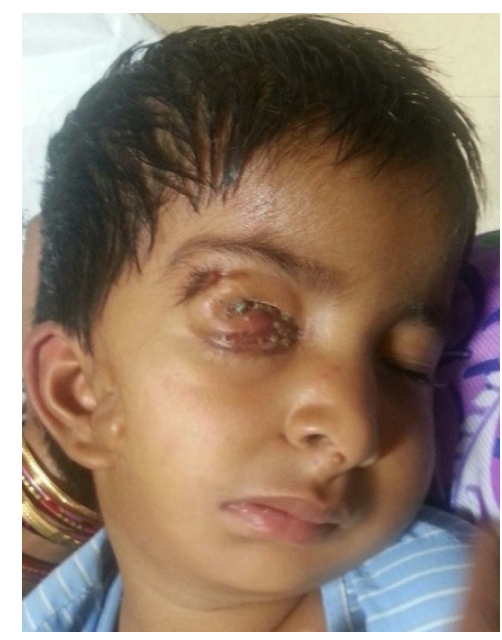

Figure 1: Facial features

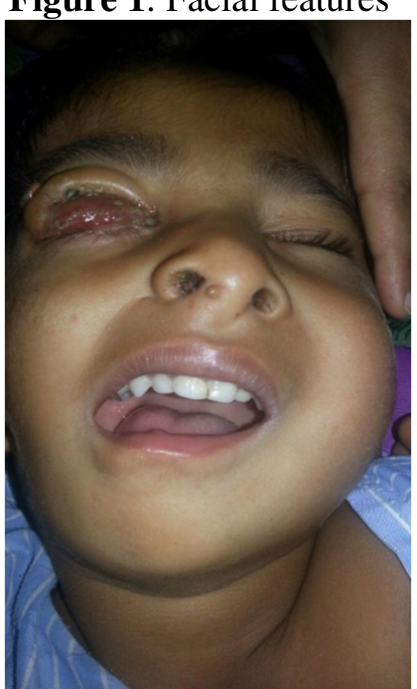

Figure 2: Airway examination (C) 2017. Kajal et al. This is an Open Access article distributed under the terms of the Creative Commons Attribution License (http: //creativecommons.org/licenses/by/4. 0), which permits unrestricted use, distribution, and reproduction in any medium, provided the original work is properly credited 
Careful airway examination revealed microsomia, high arched palate, retrognathia, deviation of mouth (Mallampati grade 3). (Figure 2) Her head and neck movements were unrestricted. She had clear chest with normal heart sounds.

Contrast Enhanced Computed Tomography paranasal sinuses showed hypoplasia of right hemi mandible with atrophied muscle of right masticator space, deformed right pinna with short right external auditory canal and occipital encephalocele (Figure 3). There was no vertebral anomaly.

In view of anticipated difficult airway we planned C-MAC guided intubation due to unavailability of fiberoptic bronchoscope in our setting. Difficult airway cart was kept ready.

In the operating room the patient was monitored with ECG, pulse oximeter, temperature probe and noninvasive blood pressure. She was induced with sevoflurane $(1 \%)$ in oxygen and i.v. atropine and fentanyl.

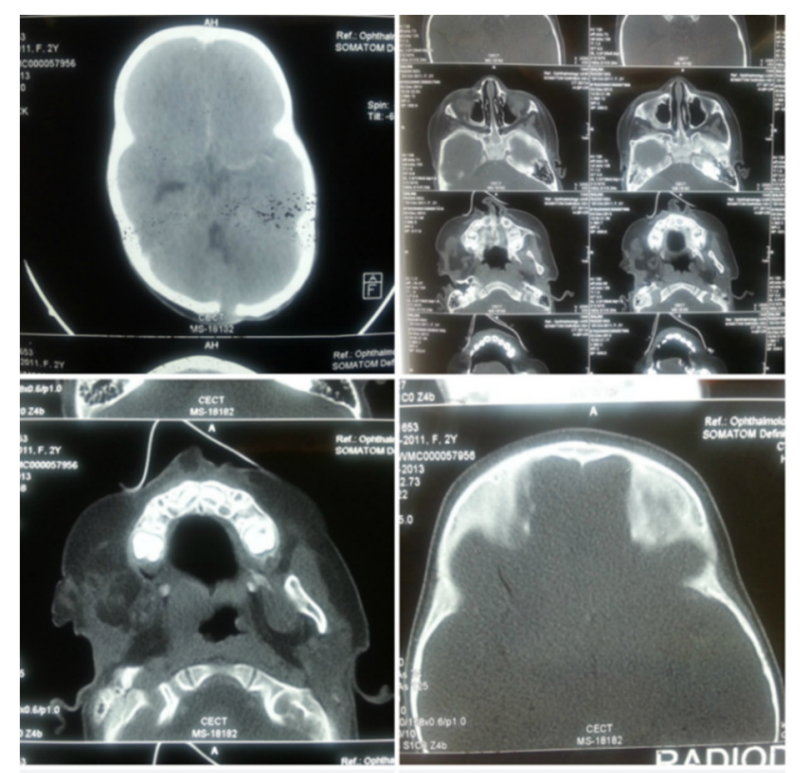

Figure 3: CECT PNS

Ensuring bag and mask ventilation, anaesthesia was deepened with sevoflurane incrementally (up to MAC 2) and intravenous propofol. Confirming adequate depth, C-MAC /Macintosh 2 blade guided laryngoscopy was performed. Only epiglottis was visualized [Cormack Lehane (CL) grade 3], intubation attempted but was unsuccessful as endotracheal tube could not be negotiated. Patient was ventilated and a second attempt with optimal external laryngeal manipulation made. This improved CL grading to 2 and patient's airway was secured with styleted $5 \mathrm{~mm}$ cuffed endotracheal tube. Intravenous atracurium was given for muscle relaxation. The anaesthesia was maintained with $2 \%$ end tidal sevoflurane in $60 \%$ nitrous oxide in oxygen and intermittent atracurium. Intravenous paracetamol was given as analgesic. Extubation and postoperative recovery were uneventful. She was discharged after 4 days.

\section{Discussion}

Goldenhar syndrome was first described by Dr. Maurice Goldenhar (1952) and later revised by Gorlin (1963). ${ }^{1}$ Literature has mentioned various devices for successful intubation in such cases as direct laryngoscopy with McIntosh blade, Magill blade, fibreoptic intubation (awake and anaesthesized condition), retrograde intubation, blind intubation and LMA. ${ }^{3-5}$ In our case microsomia, high arched palate, retrognathia, deviation of mouth and hypoplasia of right hemi mandible warranted difficult glottic visualization and introduction of laryngoscopic blade. Sugino et al used a video laryngoscope (VL) successfully in a Goldenhar child. ${ }^{6}$ We decided similarly, as it offers magnified view in real time, can be performed in neutral neck position and with reduced mouth opening. Also, role of effective external laryngeal manipulation on the oral structures could be seen in screen.

Macnair D et al showed video laryngoscopy improved CL grading and the time to intubate when compared with direct laryngoscopy. ${ }^{7}$

Among all the techniques and devices, video laryngoscope with Macintosh blade may be considered a fast, safe and effective tool for securing the airway in Goldenhar syndrome.

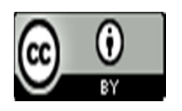

(C) 2017. Kajal et al. This is an Open Access article distributed under the terms of the Creative Commons Attribution License (http: //creativecommons.org/licenses/by/4. 0), which permits unrestricted use, distribution, and reproduction in any medium, provided the original work is properly credited 


\section{References}

1. Goldenhar M. Associations malformation de l'oeil et de l'oerielle. enparticulare le syndrome dermoideepibulbaire-appendices auriculairesfistula congenita et ses relations avec la dysotosemandibulofaciale. Journal de Genetique Humane. 1952; 1: 243-83.

2. Palmisano BW, Rusy LM (2002) Anesthesia for plastic surgery. In: Gregory GA (Ed.), Pediatric anesthesia. (4th edn.) Churchill Livingstone, New York, USA, pp. 707-745.

3. Chen PP, Cheng CK, Abdullah V, Chu CP. Tracheal intubation using suspension laryngoscopy in an infant with Goldenhar's syndrome. Anaesth Intensive Care 2001; 29(5): 548-51. PMid:11669441

4. Okuyama M, Imai M, Fujisawa E, Okuyama A, Kemmotsu O. Fiberscopic intubation under general anesthesia for children with Goldenhar syndrome. Masui 1994; 43(12): 1885-8.

PMid:7837410

5. Haxby EJ, Liban JB. Fibreoptic intubation via a laryngeal mask in an infant with Goldenhar syndrome. Anaesth Intensive Care 1995; 23(6):

753.

PMid:8669621

6. Sugino S, Kanaya N, Omote K, Namiki A. Anesthetic management in a patient with Goldenhar's syndrome using a perilaryngeal airway and a videolaryngoscope. Masui 2005; 54(12): 1367- 70.

PMid:16370341

7. Macnair D, Baraclough D, Wilson G, Bloch M, Engelhardt T. Paediatric airway management: comparing the Berci-Kaplan videolaryngoscope with direct laryngoscopy. PaediatAnesth 2009;19(6):577-80.

http://dx.doi.org/10.1111/j.1460-

9592.2009.03025.x

PMid:19645975 